typeset using JPSJ.sty <ver.1.0b $>$

\title{
Spin Fluctuation-Induced Superconductivity in $\kappa$-BEDT-TTF Compounds
}

\author{
Hisashi KONDO and Tôru MORIYA \\ Department of Physics, Faculty of Science and Technology, Science University of Tokyo, Noda 278-8510
}

(Received May 29, 2018)

\begin{abstract}
Spin fluctuation-induced superconductivity in quasi-two dimensional organic compounds, $\kappa$ BEDT-TTF salts, is investigated within a fluctuation exchange (FLEX) approximation using a half-filled Hubbard model with a right-angled isosceles triangular lattice (transfer matrices $-\tau$, $\left.-\tau^{\prime}\right)$, extending a previous work above $T_{\mathrm{c}}$. An energy gap of $\mathrm{A}_{2}$ or $\left(x^{2}-y^{2}\right)$-type develops with decreasing temperature below $T_{\mathrm{c}}$ more rapidly than in the BCS model. The calculated dynamical susceptibilities enough below $T_{\mathrm{c}}$ show sharp resonance peaks like those in certain cuprates superconductors. The calculated nuclear spin-lattice relaxation rate $1 / T_{1}$ shows a $T^{3}$ behavior below $T_{\mathrm{c}}$ in accordance with experiment. Estimated values of $1 / T_{1}$ are roughly consistent with experimental results. A prediction is made for the doping concentration dependence of $T_{\mathrm{c}}$ and the antiferromagnetic and superconductive instability points are calculated in the $U / \tau$ vs. $\tau^{\prime} / \tau$ plane.
\end{abstract}

KEYWORDS: organic superconductor, spin fluctuation, Hubbard model, triangular lattice, resonance peak, nuclear spin-lattice relaxation rate

Recent theoretical investigations indicate that the superconductivity in quasi-two dimensional organic compounds $\kappa$-(BEDT-TTF $)_{2} X$ [where $X=\mathrm{Cu}\left\{\mathrm{N}(\mathrm{CN})_{2}\right\} X^{\prime}$, $\left.X^{\prime}=\mathrm{Cl}, \mathrm{Br}\right]$ can bexplained in terms of the spin fluctuation mechanism. 1 , B, the calculated values for the transition temperature $T_{\mathrm{c}}$ as well as the d-wave character of the order parameter seem to becensistent with the existing experimental results.6. 6 ,

In a previous paper (hereafter referred to as I) we have reported the results of calculations for $T_{\mathrm{c}}$ and the normal state properties of these compounds by using a Hubbard model consisting of dimer orbitals.1. The purpose of the present communication is to report on the results of our extended calculations mainly to include the superconducting state.

The model and the approach are the same as those discussed in I. We use a half-filled Hubbard model with a right-angled isosceles triangular lattice consisting of the anti-bonding dimer orbitals with the inter-dimer transfer integrals $-\tau$ and $-\tau^{\prime}$ as shown in Fig. 1. The estimated values for the transfer integrals are $\tau \sim 0.07 \mathrm{eV}$ and $\left.\tau^{\prime} / \tau \sim 0.8 .12133\right)$ We use the fluctuation exchange (FLEX) approximation where the dynamical susceptibilities are calculated within the renormalized random phase approximation (RRPA) and the normal and the anomalous self-energies are approximated by the simplest ones including a spin fluctuation propagator without vertex corrections. This approximation has been successful in many previousinyestigations on high- $T_{\mathrm{c}}$ cuprates, etc 14, 15, 26.17, 16, 29, 20, 21) and recent studies on the vertex corrections seem to indicate that its effects will not seriously modify the FLEX results.22 (a)

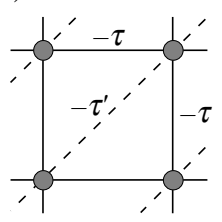

(b)

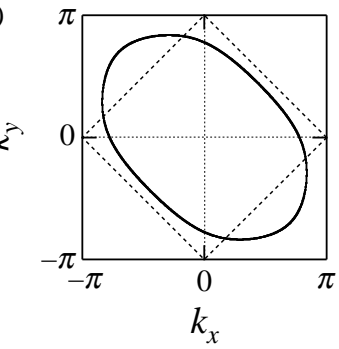

Fig. 1. (a) The model unit cell and the transfer integrals. (b) Unperturbed Fermi surface for $\tau^{\prime} / \tau=0.8$. Dashed lines show the antiferromagnetic zone boundary.

Before reporting on the results below $T_{\mathrm{c}}$ we show in Fig. 2 the calculated phase boundaries, in the $U / \tau$ vs. $\tau^{\prime} / \tau$ plane, between the paramagnetic metallic (PM) and the antiferromagnetic (AF) phases and between PM and the superconductive (SC) phases. The former is obtained from the points where the value of $1 / \chi_{Q}$ extrapolated to $T=0$ vanishes. The latter is obtained from the points of vanishing $T_{\mathrm{c}}$ calculated by extrapolating the $T_{\mathrm{c}} / \tau$ vs. $U / \tau$ plots, shown in the inset of Fig. 2, using the Padé approximants.23) This result, being consistent with the conjectures in I, shows that the AF and SC instabilities compete with each other and the latter wins for the values of $\tau^{\prime} / \tau$ larger than $\sim 0.3$. In other words, the d-wave superconductivity appears near the antiferromagnetic instability only when the electronic structure is favorable; an antiferromagnetic instability is not necessarily accompanied by a d-wave superconductivity. With increasing $U / \tau$ from a superconducting instability point we expect 


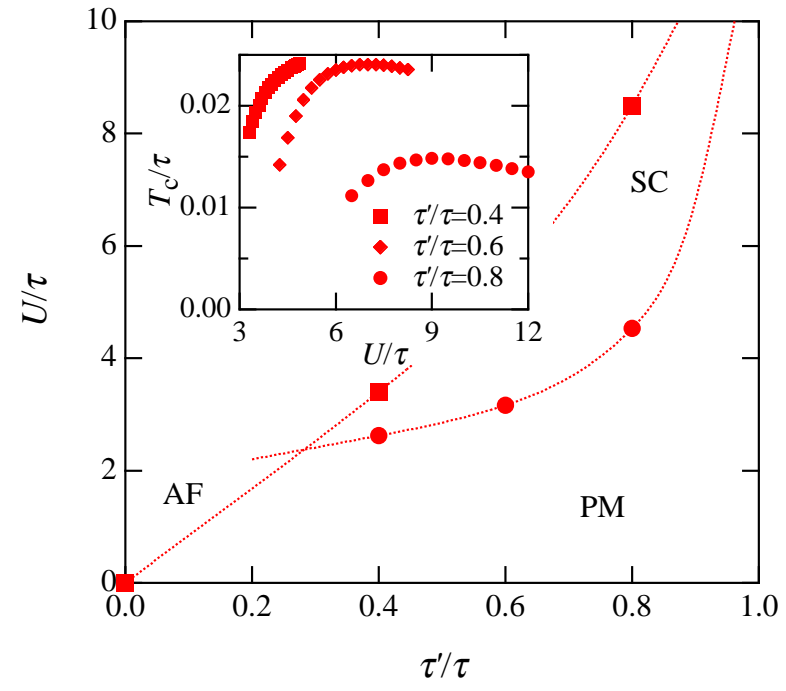

Fig. 2. Instability points of the paramagnetic state against superconductivity and antiferromagnetism. Inset shows the results of an improved calculation for $T_{\mathrm{c}} / \tau$ vs. $U / \tau$.

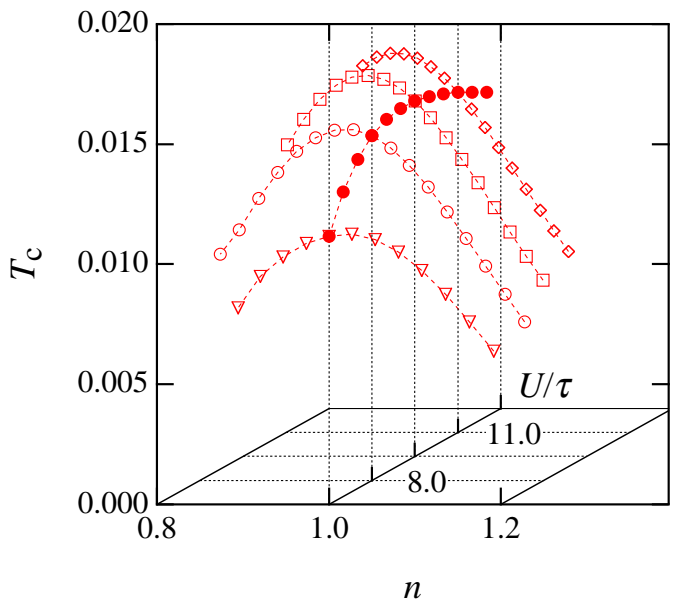

Fig. 3. Electron number (doping concentration) dependence of $T_{\mathrm{c}} / \tau$ for $\tau^{\prime} / \tau=0.8$.

a phase transition to an antiferromagnetic state. The phase boundary between the superconducting and antiferromagnetic phases must be obtained by comparing the free energies of the two phases, since the phase transition is naturally of the first order. This task is left for future studies.

Figure 3 shows the calculated values of $T_{\mathrm{c}}$ for $\tau^{\prime} / \tau=$ 0.8 for various values of $n$, the number of electrons per site, and $U / \tau$. These results may be regarded as a prediction for the doping concentration-dependence of $T_{\mathrm{c}}$ in $\kappa$-(BEDT-TTF $)_{2} X$.

Now we discuss the properties in the superconducting state. We first show in Figs. 4(a) and 4(b) the anisotropy and the temperature dependences of the gap function $\Delta_{\boldsymbol{k}}=\operatorname{Re}\left\{\Delta\left(\boldsymbol{k}, \Delta_{\boldsymbol{k}}\right)\right\}$, respectively, where the gap func-
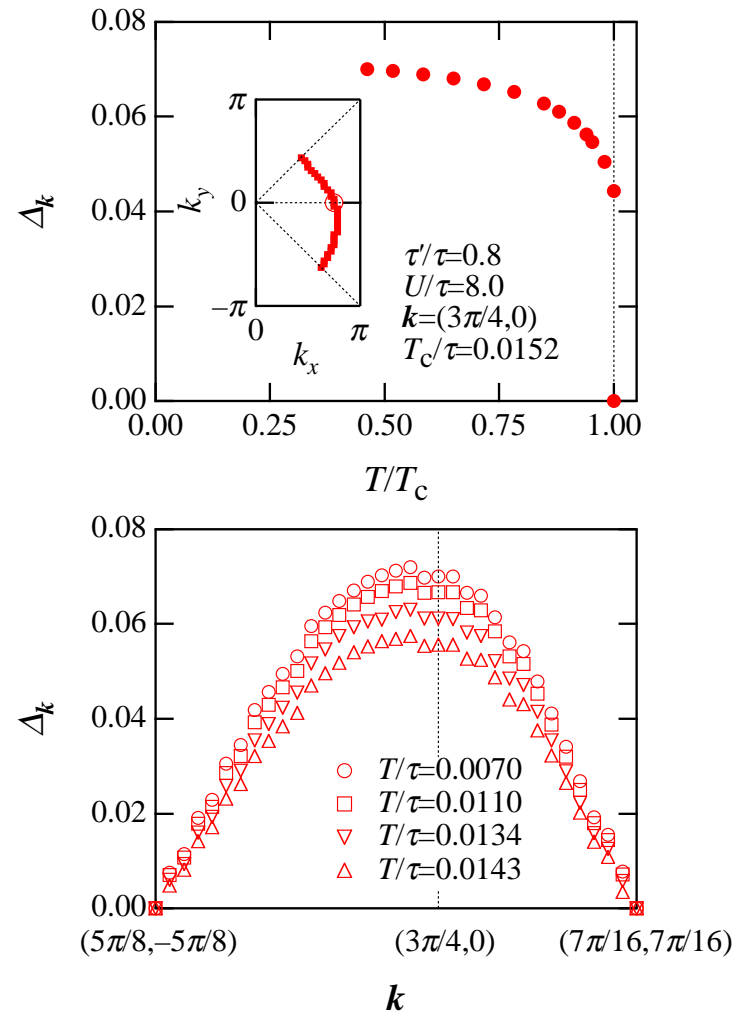

Fig. 4. Calculated gap function. (a) Temperature dependence of $\Delta_{k}$, with $k$ indicated by an open circle on the Fermi line shown in the inset. (b) Anisotropy or the wave vector-dependence of $\Delta_{\boldsymbol{k}}$, with $\boldsymbol{k}$ on the Fermi line shown in the inset of (a).

tion is defined by

$$
\begin{aligned}
\Delta(\boldsymbol{k}, \omega)= & \frac{\Sigma^{(2)}(\boldsymbol{k}, \omega+\mathrm{i} \eta)}{Z(\boldsymbol{k}, \omega)}, \\
\omega Z(\boldsymbol{k}, \omega)=\omega-\frac{1}{2}[ & \Sigma^{(1)}(\boldsymbol{k}, \omega+\mathrm{i} \eta) \\
& \left.-\Sigma^{(1)}(\boldsymbol{k},-\omega-\mathrm{i} \eta)\right],
\end{aligned}
$$

$\Sigma^{(1)}$ and $\Sigma^{(2)}$ being the normal and the anomalous selfenergies, respectively. The symmetry of the gap function is clearly of $\mathrm{A}_{2}$ or $\left(x^{2}-y^{2}\right)$-type and its amplitude develops more rapidly than in the standard BCS model below $T_{\mathrm{c}}$. Figure 5 shows the renormalized density of states at various temperatures, indicating how it is influenced by the d-wave gap formation with lowering temperature. As is expected from Fig. 4 the energy gap in the density of states develops rapidly with decreasing temperature, approaching close enough to a limiting result at around $T_{\mathrm{c}} / 2$.

The calculated dynamical susceptibilities well below $T_{\mathrm{c}}$ show strong resonance peaks around $(\pi, \pi)$ and $(\pi,-\pi)$, just as in the calculations for the high- $T_{\mathrm{c}}$ cuprates. Similarly to those in cuprates we interpret this resonance peak as a spin exciton 15,20 a bound pair of an electron and a hole excited across the energy gap. Figure 6 shows the imaginary part of the dynamical spin susceptibility 


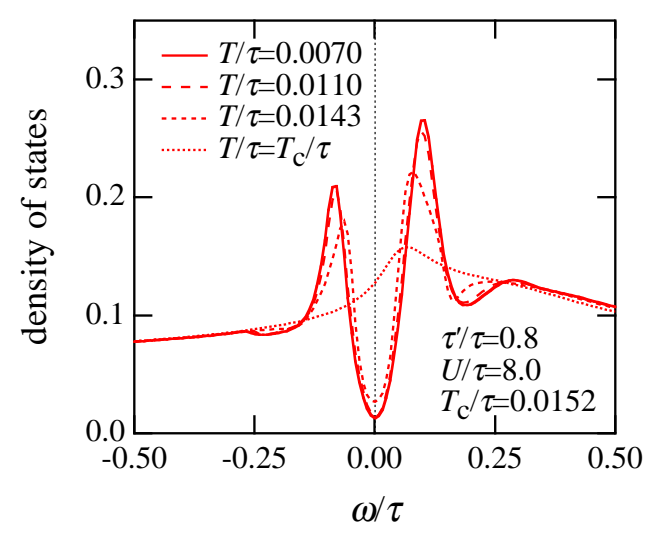

Fig. 5. Density of states at various temperatures.

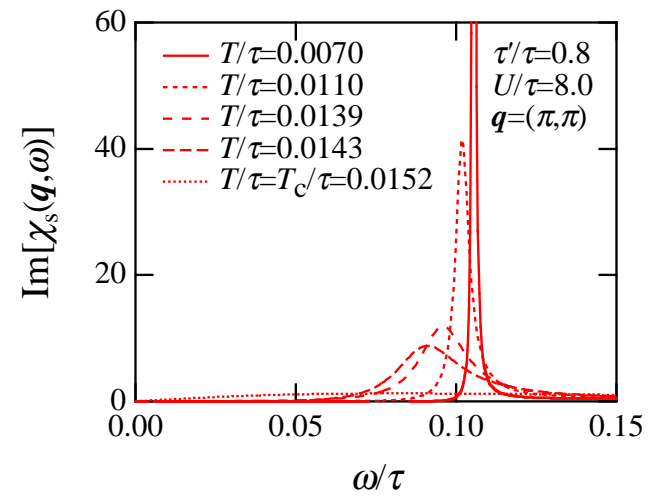

Fig. 6. Calculated imaginary part of the dynamical susceptibility for $\boldsymbol{q}=(\pi, \pi)$ at various temperatures.

at $\boldsymbol{q}=(\pi, \pi)$ at various temperatures. We see how the resonance peak develops from a broad spectrum in the normal state. Figure 7 shows the dispersion and broadening of the spin excitons. The resonance peak position $\omega_{\boldsymbol{q}}$ and the width $\gamma_{\boldsymbol{q}}$ are given by

$$
\begin{array}{r}
1-U \operatorname{Re} \bar{\chi}_{\mathrm{s}}\left(\boldsymbol{q}, \omega_{\boldsymbol{q}}\right)=0, \\
\gamma_{\boldsymbol{q}}=\frac{\operatorname{Im} \bar{\chi}_{\mathrm{s}}\left(\boldsymbol{q}, \omega_{\boldsymbol{q}}\right)}{\left\{\partial \operatorname{Re} \bar{\chi}_{\mathrm{s}}(\boldsymbol{q}, \omega) / \partial \omega\right\}_{\omega=\omega \boldsymbol{q}}},
\end{array}
$$

with

$$
\begin{aligned}
\bar{\chi}_{\mathrm{s}}\left(\boldsymbol{q}, \mathrm{i} \omega_{m}\right)= & -\frac{T}{N_{0}} \sum_{\boldsymbol{k}, n}\left[G\left(\boldsymbol{k}+\boldsymbol{q}, \mathrm{i} \omega_{n}+\mathrm{i} \omega_{m}\right) G\left(\boldsymbol{k}, \mathrm{i} \omega_{n}\right)\right. \\
& \left.+F\left(\boldsymbol{k}+\boldsymbol{q}, \mathrm{i} \omega_{n}+\mathrm{i} \omega_{m}\right) F\left(\boldsymbol{k}, \mathrm{i} \omega_{n}\right)\right],
\end{aligned}
$$

where $\omega_{m}$ and $\omega_{n}$ are the Bose and Fermi Matsubara frequencies, respectively, and $G\left(\boldsymbol{k}, \mathrm{i} \omega_{n}\right)$ and $F\left(\boldsymbol{k}, \mathrm{i} \omega_{n}\right)$ are the renormalized normal and anomalous Green's functions, respectively. The resonance peak appears only in limited regions of the $\boldsymbol{q}$-space around $(\pi, \pi)$ and $(\pi,-\pi)$. We show in Fig. 8 the temperature variation of the $\boldsymbol{q}$ integrated intensity spectrum. It is desirable to have the corresponding neutron inelastic scattering experiments on single crystals in future although such an experiment,
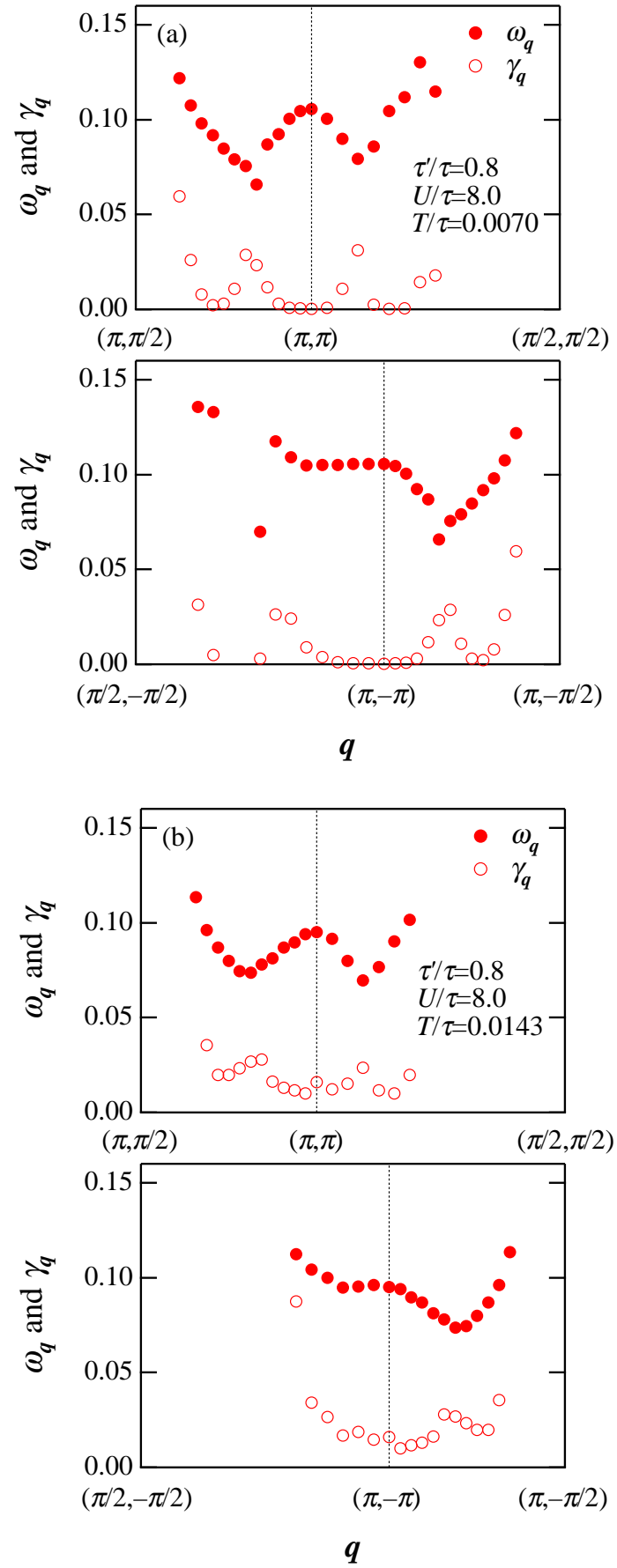

Fig. 7. Dispersion and broadening of the resonance peak or the spin excitons. (a) $T / \tau=0.0070$, (b) $T / \tau=0.0143$.

with a deuterized sample, does not seem to be very easy.

The nuclear spin-lattice relaxation rate can be calculated from the following general formula 24) using the FLEX dynamical susceptibilities:

$$
\frac{1}{T_{1}}=\frac{\gamma_{N}^{2} T}{N_{0}} \sum_{\boldsymbol{q}}\left|A_{\boldsymbol{q}}\right|^{2} \frac{\operatorname{Im} \chi^{-+}\left(\boldsymbol{q}, \omega_{0}\right)}{\omega_{0}}
$$

where $\gamma_{N}$ is the nuclear gyro-magnetic ratio, $A_{\boldsymbol{q}}$ the Fourier $\boldsymbol{q}$-component of the hyperfine coupling constant, and $\omega_{0}$ the resonance frequency. We may neglect the 


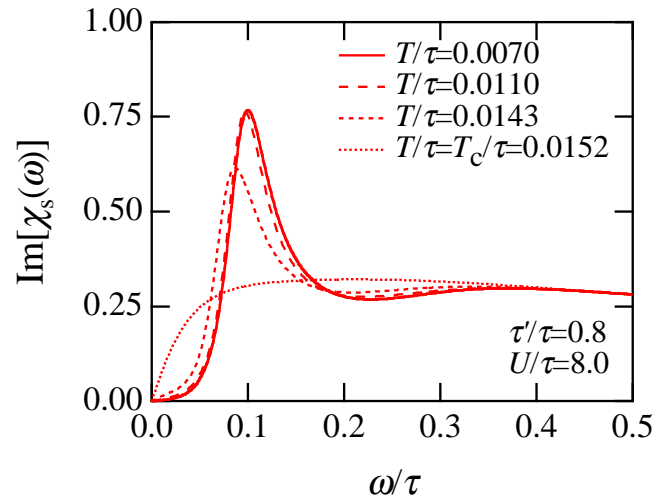

Fig. 8. $\boldsymbol{q}$-integrated spectrum of the imaginary part of the dynamical susceptibility.

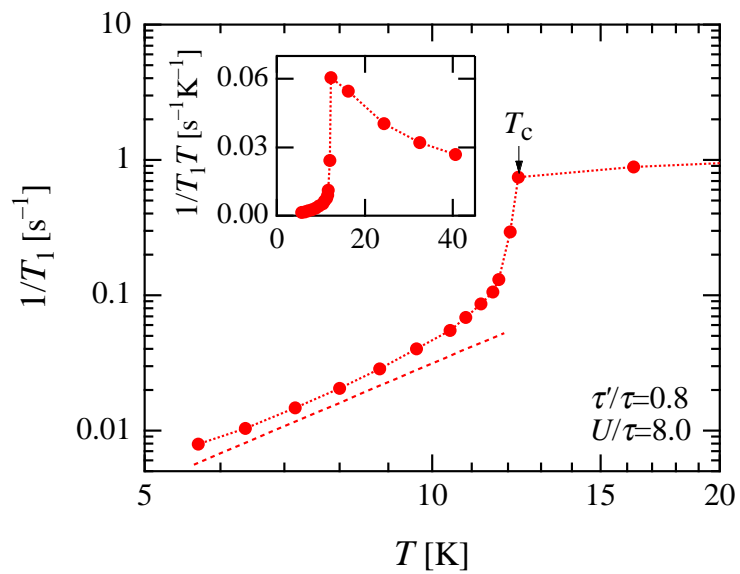

Fig. 9. Calculated nuclear spin-lattice relaxation rate $1 / T_{1}$ (logarithmic plots). The dashed line indicates a $T^{3}$ behavior. Inset shows $1 / T_{1} T$ (normal plots).

$\boldsymbol{q}$-dependence of the hyperfine coupling constant which is anisotropic at the site of ${ }^{13} \mathrm{C}$. According to ref. 8 the principal values parallel and perpendicular to the direction of the ${ }^{13} \mathrm{C}={ }^{13} \mathrm{C}$ bond are $a+2 B$ and $a-B$, respectively, with $a=1.3 \mathrm{kOe} / \mu_{\mathrm{B}}$ and $B=2.1 \mathrm{kOe} / \mu_{\mathrm{B}}$ for one spin per molecule; thus these values should be divided by 2 for one spin per dimer. For experimental results under the magnetic field parallel to the conducting layer we may approximately replace $\left|A_{\boldsymbol{q}}\right|^{2}$ in eq. (4) with $\left[(a+2 B)^{2}+(a-B)^{2}\right] / 8$, since the $\mathrm{C}=\mathrm{C}$ bond direction is nearly perpendicular to the layer. The result of calculation using this value for the coupling constant is shown in Fig. 9. The $T^{3}$ dependence of the relaxation rate below $T_{\mathrm{c}}$ as observed experimentally is well reproduced. 0 . 6 , 10 The order of magnitude seems also reasonable, in view of the approximate nature of the calculation. The observed values of $1 / T_{1} T$ above $T_{\mathrm{c}}$ has a peak at $T=T^{*} \sim 50 \mathrm{~K}$. Above this temperature $T^{*}$ the system shows insulating behavior while the system is metallic below $T^{*}$. Thus above $T^{*}$ we had better use the Heisenberg model with the Anderson superexchange.
For $T^{*}>T>T_{\mathrm{c}}$ the observed values for $1 / T_{1} T$ decrease with decreasing temperature, reminiscent of the pseudo-spin gap behaviors in high- $T_{\mathrm{c}}$ cuprates. This behavior is hard to understand within the present model and approximation. One possible explanation may be given by considering a coexistence of spin density wave (SDW) fluctuations and a charge density wave (CDW) or its slow fluctuations, although further studies are necessary before drawing any conclusion.

In summary, we have studied the spin fluctuationinduced superconducting state of quasi-two dimensional $\kappa$-(BEDT-TTF $)_{2} X @$ compounds by using a half-filled triangular Hubbard model within the FLEX approximation, extending the previous study above $T_{\mathrm{c}}$. The energy gap of $\left(x^{2}-y^{2}\right)$-type develops below $T_{\mathrm{c}}$ more rapidly than in the BCS model. Resonance peaks in the dynamical susceptibility are predicted in limited regions of the $\boldsymbol{q}$-space around $(\pi, \pi)$ and $(\pi,-\pi)$. The observed $T^{3}$ behavior of the nuclear spin-lattice relaxation rate $1 / T_{1}$ is well reproduced with a reasonable order of magnitude. We have also predicted the doping concentration dependence of $T_{\mathrm{c}}$ in these compounds. Calculations of the SC and AF instability points in the $U / \tau$ vs. $\tau^{\prime} / \tau$ plane show that the former is favorable only for $\tau^{\prime} / \tau$ larger than $\sim 0.3$, indicating that an antiferromagnetic instability dose not always accompany the d-wave superconductivity.

Finally we would like to emphasize that the present mechanism seems to be the only available mechanism to describe the anisotropic superconductivity in quasitwo dimensional $\kappa$-type BEDT-TTF compounds.25) Theoretical results seem to be generally consistent with available experimental results except for the pseudo-spin gap behavior of $1 / T_{1}$ above $\left.T_{\mathrm{c}} 26\right)$ Further experimental and theoretical investigations are desired to confirm this mechanism.

We would like to thank Dr. S. Nakamura and Dr. T. Takimoto for helpful discussions.

[1] H. Kondo and T. Moriya: J. Phys. Soc. Jpn. 67 (1998) 3695

[2] H. Kino and H. Kontani: J. Phys. Soc. Jpn. 67 (1998) 3691.

[3] J. Schmalian: Phys. Rev. Lett. 81 (1998) 4232.

[4] M. Vojta and E. Dagotto: Phys. Rev. B 59 (1999) 713.

[5] K. Kuroki and H. Aoki: cond-mat/9812026.

[6] H. Mayaffre, P. Wzietek, C. Lenoir, D. Jérome and P. Batail: Europhys. Lett. 28 (1994) 205.

[7] H. Mayaffre, P. Wzietek, D. Jérome, C. Lenoir and P. Batail: Phys. Rev. Lett. 75 (1995) 4122.

[8] A. Kawamoto, K. Miyagawa, Y. Nakazawa and K. Kanoda: Phys. Rev. Lett. 74 (1995) 3455.

[9] S. M. De Soto, C. P. Slichter, A. M. Kini, H. H. Wang, U. Geiser and J. M. Williams: Phys. Rev. B 52 (1995) 10364.

[10] K. Kanoda, K. Miyagawa, A. Kawamoto and Y. Nakazawa: Phys. Rev. B 54 (1996) 76.

[11] K. Kanoda: Physica C 282-287 (1997) 299.

[12] K. Oshima, T. Mori, H. Inokuchi, H. Urayama, H. Yamochi and G. Saito: Phys. Rev. B 38 (1988) 938.

[13] H. Kino and H. Fukuyama: J. Phys. Soc. Jpn. 65 (1996) 2158.

[14] N. E. Bickers, D. J. Scalapino and S. R. White: Phys. Rev. Lett. 62 (1989) 961.

[15] C.-H. Pao and N. E. Bickers: Phys. Rev. Lett. 72 (1994) 1870; 
Phys. Rev. B 51 (1995) 16310.

[16] P. Monthoux and D. J. Scalapino: Phys. Rev. Lett. 72 (1994) 1874.

[17] T. Dahm and L. Tewordt: Phys. Rev. B 52 (1995) 1297.

[18] M. Langer, J. Schmalian, S. Grabowski and K. H. Bennemann: Phys. Rev. Lett. 75 (1995) 4508.

[19] S. Koikegami, S. Fujimoto and K. Yamada: J. Phys. Soc. Jpn. 66 (1997) 1438.

[20] T. Takimoto and T. Moriya: J. Phys. Soc. Jpn. 66 (1997) 2459; ibid. 67 (1998) 3570.

[21] H. Kontani and K. Ueda: Phys. Rev. Lett. 80 (1998) 5619.

[22] P. Monthoux: Phys. Rev. B 55 (1997) 15261.

[23] In solving the eigen value problem to obtain $T_{\mathrm{c}}$, we took account of all the $\left(\boldsymbol{k}, \mathrm{i} \omega_{n}\right)$ points by using the Lanczos algorithm, while 1000 important points were taken into account in the previous calculation in I. New values of $T_{\mathrm{C}}$ are somewhat larger than those in I.

[24] T. Moriya: J. Phys. Soc. Jpn. 18 (1963) 516.

[25] For example, any theories based on the $t-J$ model can clearly be excluded since the half-filled electrons in these systems are in an intermediate coupling regime where an expansion in $\tau / U$ is inapplicable.

[26] Recently the temperature dependences of the electrical resistivity and the Hall coefficient have reasonably been explained with essentially the same model as the present one and within the FLEX approximation by H. Kontani and H. Kino: A talk at the PSJ meeting September 27, 1998. 